Article

\title{
Dynamic Modelling of Interactions between Microglia and Endogenous Neural Stem Cells in the Brain during a Stroke
}

\author{
Awatif Jahman Alqarni ${ }^{1,2} \mathbb{0}$, Azmin Sham Rambely ${ }^{1, * \mathbb{C}}$ and Ishak Hashim ${ }^{1}$ \\ 1 Department of Mathematical Sciences, Faculty of Science and Technology, Universiti Kebangsaan Malaysia, \\ 43600 UKM Bangi, Selangor, Malaysia; aw-aw910@hotmail.com (A.J.A.); ishak_h@ukm.edu.my (I.H.) \\ 2 Department of Mathematics, College of Sciences and Arts in Blqarn, University of Bisha, \\ Bisha 61922, Saudi Arabia \\ * Correspondence: asr@ukm.edu.my; Tel.: +60-389213244
}

Received: 26 October 2019; Accepted: 11 December 2019; Published: 16 January 2020

check for updates

\begin{abstract}
In this paper, we study the interactions between microglia and neural stem cells and the impact of these interactions on the brain cells during a stroke. Microglia cells, neural stem cells, the damage on brain cells from the stroke and the impacts these interactions have on living brain cells are considered in the design of mathematical models. The models consist of ordinary differential equations describing the effects of microglia on brain cells and the interactions between microglia and neural stem cells in the case of a stroke. Variables considered include: resident microglia, classically activated microglia, alternatively activated microglia, neural stem cells, tissue damage on cells in the brain, and the impacts these interactions have on living brain cells. The first model describes what happens in the brain at the stroke onset during the first three days without the generation of any neural stem cells. The second model studies the dynamic effect of microglia and neural stem cells on the brain cells following the generation of neural stem cells and potential recovery after this stage. We look at the stability and the instability of the models which are both studied analytically. The results show that the immune cells can help the brain by cleaning dead cells and stimulating the generation of neural stem cells; however, excessive activation may cause damage and affect the injured region. Microglia have beneficial and harmful functions after ischemic stroke. The microglia stimulate neural stem cells to generate new cells that substitute dead cells during the recovery stage but sometimes the endogenous neural stem cells are highly sensitive to inflammatory in the brain.
\end{abstract}

Keywords: chemokines; cytokines; eigenvalue stability analysis; neurogenesis; numerical solution; system of ordinary differential equations

\section{Introduction}

Strokes are diseases that affect the brain, which may cause death and disabilities in mammals [1-3]. When ischemic stroke occurs, the cells surrounding the ischemic area start to die once the outflow of blood declines below $10 \%$ of the normal blood influx [1,2]. Cells can die through necrosis or apoptosis [1,4]. Necrosis occurs very quickly after stroke onset; the necrotic cells lead to pollution of the local environment in the brain and damage the surrounding cells through laceration and the release of intracellular contents into the brain [1,4]. On the other hand, apoptosis occurs several hours or days after the stroke onset, where apoptosis occurs in the penumbra and is typically not detrimental to the neighboring cells $[1,4]$. Due to this, resident microglia are activated by the dead cells causing damage to the surrounding area, which also contributes to an increase of dead brain cells $[1,5,6]$.

Microglia are the assigned immune cells aimed at protecting brain cells from any damage $[1,2,7]$. Microglia reside within the normal brain as a ubiquitously distributed quiescent cell population 
which respond to changes within the system's micro-environment, in order to interact swiftly with diseases $[1,8,9]$. When microglia are activated their functions change, with increased capacity for phagocytosis and production of cytokines and chemokines [1]. The small secreted proteins, cytokines, which are released by the cells produce a specific effect, especially on inter-cellular interactions and communications $[1,5,6]$. When cells go through the process of phagocytosis, they are able to identify and consume large particles like pathogens, apoptotic cells, and cellular debris [10].

In living systems, free radicals and oxidants have double functions as both toxic and beneficial complex since they can be either harmful or beneficial to the body [11-13]. The activation of microglia has both beneficial and deleterious effects. The positive effects manifest through the prevention of damage extension by phagocytosis and the production of trophic molecules and anti-inflammatory cytokines, which can be conducive to tissue repair as well as neuroprotection. The harmful effects, which are toxic to tissues, occur primarily through the production of free radicals [12,13]. This reaction is important in removing damaged cells from the brain tissue; however, it can also increase the harm to brain cells by producing free radicals that are toxic to healthy cells $[2,14,15]$. There has been an increasing amount of evidence suggesting that ischemic inflammation may be vital to the issue of pathogens, as ischemic stroke results not only in damage and neuronal loss but also in sustained neuroinflammation after stroke $[3,16,17]$. Microglial cells have been shown to take part in the neural analysis associated with adult brain function responsible for the harm occurring in the brain when a stroke occurs [3]. After an ischemic stroke, a dynamic microglial polarization occurs within the harm region [18]. The microglia composition $M_{1}$ unleashes proinflammatory cytokines and oxidative mediators that prejudice living neurons, whereas the microglia composition $M_{2}$ tends to unleash neurotrophic factors, in order to stop neuronal death and promote brain repair $[17,18]$. The anti-inflammatory phenotype composition $M_{2}$ is capable of manufacturing anti-inflammatory cytokines, which are conducive to inhibiting inflammation and tissue restoration [17-19]. This shows that microglia play both positive and negative roles in stroke $[20,21]$.

In adult brains, neural stem cells (which are active throughout life) are capable of generating different cells [22,23]. Neurogenesis continues for the duration of life in the subventricular zone in all mammalian types, generating new neural stem cells during the recovery stage after ischemic stroke [24-27]. Neural stem cells appear to have many important roles, such as self-renewal and multipotency, replacement of the dead cells, and inhibition of inflammation [22,28-30]. However, endogenous neural stem cells may not be able to generate enough cells to repair the neurological damage caused by a major disease such as stroke [31]. Furthermore, the pathologic environment created after an ischemic stroke poses numerous hurdles for new neurons, which make the utilization of endogenous repair mechanisms more difficult; in particular, in directing the migration and differentiation of endogenous neural stem cells needed to repair the tissue [3,31].

Numerous mathematical models have been proposed for the dynamics of brain diseases, the functions of immune cells in terms of inflammation, and neural stem cell generation. Several models of inflammatory processes have been proposed. For example, the dynamics of inflammation from a stroke were modeled by Di Russo et al. [1] where they studied the dynamics of the densities of cells dead by necrosis and apoptosis, activated and inactivated resident microglia and the proportion of neutrophils and macrophages in the tissue. Mathematical models of inflammation proposed by the authors; Reynolds et al. [32] and Kumar et al. [33] ; for blood cells inflammation. Alharbi and Rambely [34] discussed a mathematical model of the ability of the immune system to inhibit and eliminate abnormal cells, as well as the role of dietary habits in boosting the immune system. Mathematical models for brain diseases have also been proposed by several authors $[1,35]$. For example, Hao and Friedman [35] described a model for Alzheimer's disease which utilized the microglia. Also, mathematical models have been proposed for compartmental adult neurogenesis. Nakata et al. [36] studied models of a hierarchical cell building system controlled by the mature cells. Ziebell et al. [37] introduced a mathematical model that represents different states of the adult hippocampus and the changing dynamics in stem cells during that time. Cacao and Cucinotta [38] 
developed a mathematical model of radiation-induced changes to neurogenesis, while a different study by Huang and Zhang [39] discussed the knowledge of strategies and mechanisms for neural stem cell-based therapies on brain hypoxic-ischemic injury. These studies were all focused on brain diseases and the roles of the immune system in several diseases in the blood or brain. Furthermore, some of the studies focused on the behavior of neural stem cell generation in the subventricular zone. As a starting point, we considered the model which has been recently proposed by Leah et al. [40]. We modified our models based on the model presented by Leah et al. [40], which was derived by studying the behaviors of several types of cells, microglia, brain cells, and the impact of microglial pro-inflammatory factors in living brain cells. In their model, they studied methods to represent interactions between proinflammatory and anti-inflammatory cytokines, types of microglia, and central nervous system (CNS) tissue damage, using clinical data [40].

In our model, we incorporated variables for several cytokines. Furthermore, we introduced the effect of the proinflammation by microglia on brain cells and focused on the brain during the time of stroke. However, we did not consider many types of cytokines. We studied the cytokines as parameters and their influence on the brain. Furthermore, we developed a second model to study the dynamics and stability of the interaction between the damage from microglia and the endogenous neural stem cells in the brain in the recovery stage, in order to study the ability of neural stem cells to heal the brain after stroke. The purposes of this work are to describe the biological interaction between the inflammatory cytokines from microglia and living brain cells in stroke; to study the damage on brain cells during the early stage of stroke over a 72-h duration; to study the interactions between microglia and neural stem cells and the influence of proinflammatory and anti-inflammatory cytokines on brain cells; to analyze the dynamic effect of microglia by stimulating the generation and the proliferation of neural stem cells during the recovery phase after the stroke; and to draw conclusions about the dynamics of neural stem cells to improve the brain after a stroke within a mathematical framework. Our final aim is to understand the positive and negative aspects of this biological process, which could be helpful for the development of therapeutic methods using endogenous neural stem cells in ischemic stroke, by studying the stability of the models.

In Section 2, we present a model—called stroke-microglia-damage (SMD)—of the effect of microglia on the brain during stroke onset, and another model-called stroke-microglia-neural stem cells-recovery (SMNR) — of the interaction between the microglia and neural stem cells and their impact on the brain during a stroke, including analysis of the equilibrium points of the models and their stability states. Numerical experiments of the modified models are detailed in Section 3. Finally, Section 4 presents the conclusions of the study.

\section{Mathematical Models}

We modified mathematical models of biological processes to investigate the impact of microglia and neural stem cells on the brain by increasing damage or assisting it in the recovery stage in two steps, illustrated by systems of ordinary differential equations. We developed the SMD model based on a model presented by Leah et al. [40], who proposed a mathematical model for neuroinflammation in traumatic brain injury using mathematical modeling with clinical data. The system of ordinary differential equations they derived described the dynamics of biological processes for multiple interactions post-injury. The first model, called the SMD model, that we shall develop describes the roles of microglia in the first $72 \mathrm{~h}$ after stroke onset without any generation of neural stem cells, while the second model, called the SMNR model, describes the state from the third day when neural stem cells start to generate. The resting microglia are the immune cells, $M_{0}$, which reside in the brain, and will be activated when a stroke occurs to a classic proinflammatory $M_{1}$ or an alternative anti-inflammatory $M_{2}$ state, where the resting microglia polarizes into two activation states, $M_{1}$ or $M_{2}$, in reaction to the dead cells from ischemia [18,19]. The cytokines signal for the activation of resting microglia into two phenotypes, proinflammation $M_{1}$, and anti-inflammation $M_{2}$, microglia denoted by $R_{1}$ and $R_{2}$, respectively. When microglia cells are activated in stroke, they become either $M_{1}$ or $M_{2}$ 
phenotypes. At the start of the stroke, the microglia are biased towards the $M_{1}$ phenotype rather than $M_{2}$ phenotype, since $R_{1}>R_{2}$. We assume that in stroke onset with this bias towards $M_{1}$, the transition rate from $M_{1}$ into $M_{2}$ phenotype is very small, approximately equal to zero in the first $60 \mathrm{~h}$ after stroke onset [1], and the rate of anti-inflammation cytokines, $R_{2}$, is at a smaller rate than the rate of proinflammation cytokines, $R_{1}$, in the SMD model. We take $R_{1}<R_{2}$ when the rate of proinflammation cytokines, $R_{1}$, is at a lower rate than the rate of anti-inflammation cytokines, $R_{2}$ in the recovery stage in the SMNR model. Neural stem cells in the subventricular zone from the adult mammalian brain give rise to neuroblasts, which migrate to the injury region and generate new cells [22,28,41]. Microglia have a positive and negative impact on generating neural stem cells [42]. $M_{1}$ can impair neural stem cells [18], while $M_{2}$ shows the ability to stimulate the generation and the proliferation of neural stem cells $N_{s c}$ Ref. [19]. We focus our model on the mature neural stem cells only in the SMNR because no generation of neural stem cells occurs in this stage of stroke onset.

\subsection{Modelling of the Effect of Microglia on the Brain in a Stroke Onset (SMD)}

In this study, we modified the dynamic system of the SMD model based on the model presented in [40], which illustrates the behavior of the impact of activated microglia on brain cells in the $72 \mathrm{~h}$ following a stroke. We consider a non-linear system of ordinary differential equations, which describes the effects of microglia $M_{0}(t), M_{1}(t), M_{2}(t)$, and the damage from microglia, $D(t)$, on brain cells, $C(t)$, in the $72 \mathrm{~h}$ after stroke onset as follows:

$$
\frac{d M_{0}}{d t}=\alpha-\left[R_{1}+R_{2}+\mu\right] M_{0}(t) .
$$

This equation demonstrates that the microglia are activated after an ischemic stroke and are polarized either towards a classic state, $M_{1}$, by proinflammatory cytokines, leading to an adaptive immune response and causing additional neuronal damage; or towards an alternative state, $M_{2}$, which is the anti-inflammatory phenotype induced by anti-inflammatory cytokines, which is thought to inhibit inflammation and enhance tissue repair $[18,19,40]$. Although we do not specify the types of cytokines in our models, we focus on the influences of the cytokines which gather in the region of damage; which are determined to either increase $M_{1}$ polarization or to change the microglia into the $M_{2}$ state. For mathematical modeling purposes, we assume that resting microglia $M_{0}$ are generated at a constant rate $(\alpha)$ and die at a constant rate $(\mu)$. The function of the activated microglia is to clean up dead cells produced by ischemia and cytokines caused by dead cells [43]. Microglia are activated by the cytokines caused by dead cells: the $M_{1}$ phenotype is induced by proinflammation cytokine signals $R_{1}$ and the $M_{2}$ phenotype is induced by anti-inflammation cytokine signals $R_{2}[5,17,43]$. Furthermore, a shift from $M_{1}$ to $M_{2}$ may be induced by $R_{3}$ signaling [5], where $R_{3}$ is the result of a transition from proinflammatory to anti-inflammatory cytokines. This transition only appears in the SMNR model, occurring $60 \mathrm{~h}$ after the stroke onset [1]. The following differential equations illustrate the behavior of the activated microglia $M_{1}$ and $M_{2}$ :

$$
\begin{aligned}
& \frac{d M_{1}}{d t}=R_{1} M_{0}(t)-\left[\delta D(t)+\gamma_{1}\right] M_{1}(t), \\
& \frac{d M_{2}}{d t}=R_{2} M_{0}(t)-\gamma_{2} M_{2}(t),
\end{aligned}
$$

where $R_{1}$ represents the rate of $M_{1}$ activation, $R_{2}$ is the rate of $M_{2}$ activation, $\delta$ is the rate of damage by $M_{1}$ activation, $\gamma_{1}$ is the death rate of $M_{1}$, and $\gamma_{2}$ is the death rate of $M_{2}$. The $M_{1}$ phenotype is essential for cell recovery, due to their protection mechanisms against the damage which recruits immune cells to the region of injury. The $M_{2}$ phenotype microglia also play a role in reducing damage, by clearing the brain of dead cells and assisting in neurogenesis, as well as inhibiting inflammation. On the other hand, the microglia can also play a negative role, through secretion of damaging proinflammatory cytokines which increase inflammation in the area of healthy cells $[5,17]$. We consider the damage $D$ caused 
by the activated microglia to represent secondary damage in the case of stroke [2,14]. The following differential equation describes this damage:

$$
\frac{d D}{d t}=D(t)\left[M_{1}(t)\left(\delta-r_{1}\right)-\beta_{1} C(t)-r_{2} M_{2}(t)\right],
$$

where $\beta_{1}$ denotes the rate of effect of damage on brain cells, $r_{1}$ denotes the rate of damage clearance by $M_{1}$, and $r_{2}$ denotes the rate of damage clearance by $M_{2}$. In our model, we use the impact of the damage by microglia on the living brain cells, $C$. The following ordinary differential equation describes this effect on brain cells:

$$
\frac{d C}{d t}=C(t)\left[\beta_{1} D(t)-\beta_{0}\right]
$$

where $\beta_{0}$ is the rate of cells dying from ischemic stroke.

Thus, the SMD model is expressed as follows:

$$
\begin{aligned}
\frac{d M_{0}}{d t} & =\alpha-\left[R_{1}+R_{2}+\mu\right] M_{0}(t), \\
\frac{d M_{1}}{d t} & =R_{1} M_{0}(t)-\left[\delta D(t)+\gamma_{1}\right] M_{1}(t), \\
\frac{d M_{2}}{d t} & =R_{2} M_{0}(t)-\gamma_{2} M_{2}(t), \\
\frac{d D}{d t} & =D(t)\left[M_{1}(t)\left(\delta-r_{1}\right)-\beta_{1} C(t)-r_{2} M_{2}(t)\right], \\
\frac{d C}{d t} & =C(t)\left[\beta_{1} D(t)-\beta_{0}\right],
\end{aligned}
$$

with initial values $M_{0}(0)=1$ [1], $M_{1}(0)=0.1514$ [1], $M_{2}(0)=0.02$ [35], $D(0)=0.4$ [1], and $C(0)=0.28$ [35]. Furthermore, we assumed that, in steady state, $M_{1}>M_{2}$ for $72 \mathrm{~h}$ and that neural stem cells are not generated.

\subsubsection{Equilibrium Points for the SMD Model}

In this section, we calculate the equilibrium points of the system and determine the parameters for the existence of different types of biological states. We now determine the steady-state solutions as follows:

$$
\frac{d M_{0}}{d t}=\frac{d M_{1}}{d t}=\frac{d M_{2}}{d t}=\frac{d D}{d t}=\frac{d C}{d t}=0 .
$$

Equilibrium points are stable if they remain constant over time or continually balance change in one direction by that in another. We classify three steady states as follows:

Definition 1 (State of the activation of microglia). We define the microglia that reside in a healthy brain as the absence of a high activation for these cells in the brain when any damage occurs in the region. The steady-state of the form $M_{0} ; M_{1} ; M_{2}>0$ and $D, C=0$ indicates that the function of immune cells is normal and the microglia do not cause any damage in the brain.

Definition 2 (State of the beginning of damage from activated microglia $M_{1}$ ). We define the beginning of the damage by the increased rate of proinflammation cytokines where the existence of high activation of microglia will cause damage to the living brain cells. The steady-state of the form $M_{0} ; M_{1} ; M_{2} ; D>0, C=0$ indicates the beginning activation of proinflammation from $M_{1}$ microglia. 
Definition 3 (State of the damage from activated microglia in brain cells). We define the impact of the damage caused by microglia on the living brain cells. The steady state of the form $M_{0} ; M_{1} ; M_{2} ; D ; C>0$ indicates damage of the living brain cells by active microglia.

Populations of microglia cells $\left(M_{0}, M_{1}, M_{2}\right)$, the damage on the tissue by proinflammation, $D$ and the impact of this damage on the living brain cells in the CNS, $C$ are positive or equal to zero for all equations. Thus, we obtain the equilibrium points by solving system (1)-(5) to determine the positive equilibrium points if and only if $M_{0}, M_{1}, M_{2}, D$ and $C$ exemplify the positive solutions.

Proposition 1 (Nonnegative Equilibrium for the SMD model). We assume the equilibrium points for $S M D$ system, $M_{0} ; M_{1} ; M_{2} ; D ; C>0$ satisfy the following conditions:

- $\delta>r_{1}$

- $R_{1} \gamma_{2}\left(-r_{1}+\delta\right)>r_{2} R_{2} \gamma_{1}$

- $\alpha R_{1} \beta_{1} \gamma_{2}\left(-r_{1}+\delta\right)>r_{2} R_{2}\left(\beta_{1} \gamma_{1}+\beta_{0} \delta\right)$.

Then and only then can there exist nonnegative real steady states.

Proof. The proof can be clearly found from Definition 1, 2 and 3.

Hence, the equilibrium point is given as follows:

The equilibrium point for the activation of microglia, $E_{r}$

Corollary 1. The equilibrium point $E_{r}=\left(M_{0}, M_{1}, M_{2}, 0,0\right)$ exists in $\Re_{+}^{5}$ if and only if $M_{0}, M_{1}, M_{2}$ are the nonnegative roots and $D=0, C=0$. This point is given by

$$
E_{r}=\left(M_{0}, M_{1}, M_{2}, 0,0\right)=\left(\frac{\alpha}{x}, \frac{\alpha R_{1}}{\gamma_{1} x}, \frac{R_{2} \alpha}{\gamma_{2} x}, 0,0\right)
$$

where,

$$
x=R_{1}+R_{2}+\mu
$$

Thus, in the normal state in mammalian brains, we obtain a normal situation when there is no activation of microglia on the brain where $D, C=0$ given that there is no damage on the brain cells, where the equilibrium point $E_{r}$ indicated the activation of microglia steady state.

The equilibrium point for high activation of microglia, $E_{p}$

Corollary 2. The equilibrium point $E_{p}$ exists in $\Re_{+}^{5}$ if and only if, $M_{0}, M_{1}, M_{2}$ and $D$ are the nonnegative roots and $C=0$. Then, the equilibrium point of proinflammation microglia through $72 \mathrm{~h}$ after stroke is given as follows:

$$
E_{p}=\left(\frac{\alpha}{x}, \frac{r_{2} R_{2} \alpha}{\gamma_{2}\left(-r_{1}+\delta\right) x}, \frac{R_{2} \alpha}{\gamma_{2} x}, \frac{-r_{2} R_{2} \gamma_{1}+R_{1} \gamma_{2}\left(-r_{1}+\delta\right)}{r_{2} R_{2} \delta}, 0\right)
$$

The model has an equilibrium point for the activation of microglia. During this stage, $M_{1}$ has a significant impact. The equilibrium point $E_{p}$ describes the activation by cytokines which obtains higher proinflammation from $R_{1}$ than the rate of the second kind of cytokines $R_{2}$ if and only if the rate of damage on the living brain cells by $M_{1}$ is more than $r_{1}$, which is the clearance damage by the immune cells microglia $M_{1}$.

The equilibrium points for the impact of proinflammation microglia $M_{1}$ on brain cells, $E_{d}$ 
Corollary 3. The equilibrium point $E_{d}$ exists in $\Re_{+}^{5}$ if and only if $M_{0}, M_{1}, M_{2}, D$ and $C$ are the nonnegative roots. Then, the equilibrium point $E_{d}$ has an impact on the damage to the living brain cells through $72 \mathrm{~h}$ after stroke onset given as follows:

$$
E_{d}=\left(\frac{\alpha}{x}, \frac{R_{1} \beta_{1} \alpha}{\kappa x}, \frac{R_{2} \alpha}{\gamma_{2} x}, \frac{\beta_{0}}{\beta_{1}}, \frac{\left(R_{1} \beta_{1} \gamma_{2}\left(-r_{1}+\delta\right)-r_{2} R_{2} \kappa\right) \alpha}{\beta_{1} \gamma_{2} \kappa x}\right),
$$

where,

$$
\kappa=\left(\beta_{1} \gamma_{1}+\beta_{0} \delta\right)
$$

\subsubsection{Stability of Equilibrium Points}

We now study the stability of equilibrium points for the effect of microglia on brain cells for $72 \mathrm{~h}$ without neural stem cells by using definitions $1-3$. For the eigenvalues associated without neural stem cell equilibrium in stroke onset, the $(5 \times 5)$ Jacobian matrix of the system $(1)-(5)$ is given by

$$
J_{1}=\left[\begin{array}{ccccc}
-x & 0 & 0 & 0 & 0 \\
R_{1} & -\gamma_{1}-D \delta & 0 & -M_{1} \delta & 0 \\
R_{2} & 0 & -\gamma_{2} & 0 & 0 \\
0 & D\left(-r_{1}+\delta\right) & -D r_{2} & -M_{2} r_{2}-c \beta_{1}+M_{1}\left(-r_{1}+\delta\right) & -D \beta_{1} \\
0 & 0 & 0 & c \beta_{1} & D \beta_{1}-\beta_{0}
\end{array}\right] .
$$

1. Stability analysis of equilibrium point, $E_{r}$ :

Theorem 1. Suppose that the function $f: \Gamma \rightarrow \Re_{+}^{5}$ where $\Gamma$ is a domain in $\Re_{+}^{5}$, and suppose that $E_{r}=\left(M_{0}, M_{1}, M_{2}, 0,0\right) \in \Gamma$ is an equilibrium point at which at least one eigenvalue of the Jacobian matrix has a positive real part. Then, $E_{r}$ is an unstable equilibrium point of $f$.

Proof. The Jacobian $J_{1}$ at equilibrium point $E_{r}$ is calculated as follows:

$$
J\left[E_{r}\right]=\left[\begin{array}{ccccc}
-x & 0 & 0 & 0 & 0 \\
R_{1} & -\gamma_{1} & 0 & a_{24} & 0 \\
R_{2} & 0 & -\gamma_{2} & 0 & 0 \\
0 & 0 & 0 & a_{44} & 0 \\
0 & 0 & 0 & 0 & -\beta_{0}
\end{array}\right]
$$

where,

$$
a_{24}=\frac{-R_{1} \alpha \delta}{\gamma_{1} x}, a_{44}=\frac{-r_{2} R_{2} \alpha}{\gamma_{2} x}+\frac{R_{1} \alpha\left(-r_{1}+\delta\right)}{\gamma_{1} x} .
$$

The characteristic equation for the Jacobian $J\left[E_{r}\right]$ is given by

$$
\left(\beta_{0}+\lambda\right)\left(\gamma_{2}+\lambda\right)\left(\gamma_{1}+\lambda\right)(x+\lambda)(\lambda+y)=0
$$

We assume that $x=R_{1}+R_{2}+\mu, y=\frac{r_{2} R_{2} \alpha}{\gamma_{2} x}+\frac{R_{1} \alpha\left(r_{1}-\delta\right)}{\gamma_{1} x}$.

Then, the eigenvalues of Jacobian matrix $J\left[E_{r}\right]$ are given by:

$$
\begin{aligned}
& \lambda_{1}=-x<0, \quad \lambda_{2}=-\beta_{0}<0, \\
& \lambda_{3}=-\gamma_{1}<0, \quad \lambda_{4}=-\gamma_{2}<0, \quad \lambda_{5}=-y=\frac{\alpha\left(-r_{2} R_{2} \gamma_{1}+R_{1} \gamma_{2}\left(-r_{1}+\delta\right)\right)}{\left(\gamma_{1} \gamma_{2} x\right)}>0 .
\end{aligned}
$$


The eigenvalues $\lambda_{1}, \ldots, \lambda_{4}$ are negative, but $\lambda_{5}$ is positive. Therefore, $E_{r}$ is an unstable equilibrium point.

2. Stability Analysis of Equilibrium point, $E_{p}$ :

Theorem 2. Suppose that the function $f: \Gamma \rightarrow \Re_{+}^{5}$ where $\Gamma$ is a domain in $\Re_{+}^{5}$, and suppose that $E_{p}=\left(M_{0}, M_{1}, M_{2}, D, 0\right) \in \Gamma$ is an equilibrium point at which at least one eigenvalue of the Jacobian matrix has a positive real part and $D>0, C=0$. Then, $E_{p}$ is an unstable equilibrium point of $f$.

Proof. The Jacobian $J_{1}$, at $E_{p}$ calculated as:

$$
J\left[E_{p}\right]=\left[\begin{array}{ccccc}
-x & 0 & 0 & 0 & 0 \\
R_{1} & b_{22} & 0 & b_{24} & 0 \\
R_{2} & 0 & -\gamma_{2} & 0 & 0 \\
0 & b_{42} & b_{43} & b_{44} & b_{45} \\
0 & 0 & 0 & 0 & b_{55}
\end{array}\right],
$$

where,

$$
\begin{aligned}
& b_{22}=-\gamma_{1}-\frac{\left(-r_{2} R_{2} \gamma_{1}+R_{1} \gamma_{2}\left(-r_{1}+\delta\right)\right)}{r_{2} R_{2}}<0, \quad b_{24}=\frac{r_{2} R_{2} \alpha \delta}{\gamma_{2}\left(r_{1}-\delta\right) x}<0, \\
& b_{42}=\frac{\left(-r_{1}+\delta\right)\left(-r_{2} R_{2} \gamma_{1}+R_{1} \gamma_{2}\left(-r_{1}+\delta\right)\right)}{r_{2} R_{2} \delta}>0, \quad b_{43}=\frac{-\left(-r_{2} R_{2} \gamma_{1}+R_{1} \gamma_{2}\left(-r_{1}+\delta\right)\right)}{R_{2} \delta}, \\
& b_{44}=\frac{-r_{2} R_{2} \alpha}{\gamma_{2} x}-\frac{r_{2} R_{2} \alpha\left(-r_{1}+\delta\right)}{\gamma_{2}\left(r_{1}-\delta\right) x}<0, \quad b_{45}=-\frac{\beta_{1}\left(-r_{2} R_{2} \gamma_{1}+R_{1} \gamma_{2}\left(-r_{1}+\delta\right)\right)}{r_{2} R_{2} \delta}, \\
& b_{55}=\frac{R_{1} \beta_{1} \gamma_{2}\left(-r_{1}+\delta\right)-r_{2} R_{2}\left(\beta_{1} \gamma_{1}+\beta_{0} \delta\right)}{r_{2} R_{2} \delta}>0 .
\end{aligned}
$$

From the Jacobian $J\left[E_{p}\right]$, the characteristic equation is given by

$$
\left(\gamma_{2}+\lambda\right)\left(b_{55}-\lambda\right)(x+\lambda)\left(q_{2} \lambda^{2}+q_{1} \lambda^{1}+q_{0}\right)=0,
$$

where,

$$
\begin{aligned}
q_{2} & =r_{2} R_{2} \gamma_{2}\left(r_{1}-\delta\right) x, q_{1}=-R_{1} \gamma_{2}^{2}\left(r_{1}-\delta\right)^{2} x, \\
q_{0} & =-r_{2} R_{2} \alpha\left(r_{2} R_{2} \gamma_{1}+R_{1} \gamma_{2}\left(r_{1}-\delta\right)\right)\left(r_{1}-\delta\right), \\
x & =R_{1}+R_{2}+\mu .
\end{aligned}
$$

By Proposition 1 one of the eigenvalues is positive. So, $J\left(E_{p}\right)$ has one at least positive root, which indicates that the equilibrium point $E_{p}$ is unstable [44].

3. Stability analysis of equilibrium point, $E_{d}$ :

Theorem 3. Suppose that the function $f: \Gamma \rightarrow \Re_{+}^{5}$ where $\Gamma$ is a domain in $\Re_{+}^{5}$, and suppose that $E_{d}=\left(M_{0}, M_{1}, M_{2}, D, C\right) \in \Gamma$ is an equilibrium point where all the eigenvalues of the Jacobian matrix have negative real parts at the equilibrium point $E_{d}$ and $D>0, C>0$. Then, $E_{d}$ is a stable equilibrium point of $f$. 
Proof. The Jacobian $J_{1}$ at $E_{d}$ is calculated as follows:

$$
J\left[E_{d}\right]=\left[\begin{array}{ccccc}
-x & 0 & 0 & 0 & 0 \\
R_{1} & c_{22} & 0 & c_{24} & 0 \\
R_{2} & 0 & -\gamma_{2} & 0 & 0 \\
0 & c_{42} & c_{43} & c_{44} & -\beta_{0} \\
0 & 0 & 0 & c_{54} & 0
\end{array}\right],
$$

where,

$$
\begin{aligned}
c_{22} & =-\gamma_{1}-\frac{\beta_{0} \delta}{\beta_{1}}<0, \quad c_{24}=-\frac{R_{1} \alpha \beta_{1} \delta}{\left(\beta_{1} \gamma_{1}+\beta_{0} \delta\right) x}<0, \\
c_{42} & =\frac{\beta_{0}\left(-r_{1}+\delta\right)}{\beta_{1}}>0, \quad c_{43}=-\frac{r_{2} \beta_{0}}{\beta_{1}}<0, \\
c_{44} & =-\frac{r_{2} R_{2} \alpha}{\gamma_{2} x}+\frac{\left.R_{1} \alpha \beta_{1}\left(-r_{1}+\delta\right)\right)}{\left(\beta_{1} \gamma_{1}+\beta_{0} \delta\right) x} \\
& +\frac{\alpha\left(R_{1} \beta_{1} \gamma_{2}\left(r_{1}-\delta\right)+r_{2} R_{2}\left(\beta_{1} \gamma_{1}+\beta_{0} \delta\right)\right)}{\gamma_{2}\left(\beta_{1} \gamma_{1}+\beta_{0} \delta\right) x}>0, c_{45}=-\beta_{0}<0 \\
c_{54} & =-\frac{\alpha\left(R_{1} \beta_{1} \gamma_{2}\left(r_{1}-\delta\right)+r_{2} R_{2}\left(\beta_{1} \gamma_{1}+\beta_{0} \delta\right)\right)}{\gamma_{2}\left(\beta_{1} \gamma_{1}+\beta_{0} \delta\right) x}>0 .
\end{aligned}
$$

The characteristic equation is given by

$$
\left(\gamma_{2}+\lambda\right)(x+\lambda)\left(N_{3} \lambda^{3}+N_{2} \lambda^{2}+N_{1} \lambda+N_{0}\right)=0
$$

Thus, we can find the first two eigenvalues directly:

$$
\lambda_{1}=-\gamma_{2}, \lambda_{2}=-x
$$

Here, we can apply the Routh-Hurwitz Criterion if and only if [45]:

- $\quad N_{2}>0$,

- $\quad N_{0}>0$,

- $\quad N_{2} N_{1}>N_{0} N_{3}$.

where

$$
\begin{aligned}
& N_{0}=\alpha \beta_{1} \beta_{0}\left(\beta_{1} \gamma_{1}+\beta_{0} \delta\right)\left(R_{1} \beta_{1} \gamma_{2}\left(-r_{1}+\delta\right)-r_{2} R_{2}\left(\beta_{1} \gamma_{1}+\beta_{0} \delta\right)\right)>0, \\
& N_{1}=\alpha \beta_{1}^{2} \beta_{0}\left(R_{1} \gamma_{2}\left(-r_{1}+\delta\right)\left(\beta_{1}+\delta\right)-r_{2} R_{2}\left(\beta_{1} \gamma_{1}+\beta_{0} \delta\right)\right)>0, \\
& N_{2}=\beta_{1} \gamma_{2}\left(\beta_{1} \gamma_{1}+\beta_{0} \delta\right)^{2} x>0, N_{3}=\beta_{1}^{2} \gamma_{2}\left(\beta_{1} \gamma_{1}+\beta_{0} \delta\right) x>0 .
\end{aligned}
$$

Since from Proposition 1,

$$
N_{2} N_{1}-N_{0} N_{3}=R_{1} \alpha \beta_{1}^{3} \beta_{0} \gamma_{2}^{2} \delta\left(-r_{1}+\delta\right)\left(\beta_{1} \gamma_{1}+\beta_{0} \delta\right)^{2} x>0
$$

Then, $N_{2}, N_{0}>0$ and $N_{2} N_{1}-N_{0} N_{3}>0$, 
Now, we apply the Routh-Hurwitz theorem for $N_{3} \lambda^{3}+N_{2} \lambda^{2}+N_{1} \lambda+N_{0}=0$, giving

$$
\left|\begin{array}{ccc}
\lambda^{3} & N_{3} & N_{1} \\
\lambda^{2} & N_{2} & N_{0} \\
\lambda^{1} & N^{*} & 0 \\
\lambda^{0} & N_{0} & 0
\end{array}\right|,
$$

where,

$$
N^{*}=\frac{N_{2} N_{1}-N_{3} N_{0}}{N_{2}}
$$

thus,

$$
N^{*}=R_{1} \alpha \beta_{1}^{2} \beta_{0} \gamma_{2} \delta\left(-r_{1}+\delta\right)>0 .
$$

Since all the coefficients in the first column have positive signs; the Equation (10) has no roots with positive real parts and two of the eigenvalues are negative; thus, the equilibrium point $E_{d}$ is stable. Activated microglia are capable of cleaning dead cells; however, they produce free radicals from brain cells, which increases the damage in brain cells during a stroke. This lead to further death of brain cells [17,32].

Remark 1. The effect of microglia on brain cells in a stroke (which includes activated microglia at stroke onset) on the dynamic system of SMD model can be deduced, as follows:

- As a result of Theorem 1 and Definition 1, the damage, $D$, can invade the SMD system if $\lambda_{5}>0$.

- As a result of Theorem 2 and Definition 2, this means that the damage, $D>0$, invades $C$.

- As a result of Theorem 3 and Definition 3, this means that the damage, $D>0$, causes the death of $C$.

- The SMD model is stable when the brain cells are affected by the proinflammatory cytokines of microglia; however, when the rate of production of proinflammatory cytokines leads to an increase in damage, the possibility of death of the brain cells is introduced.

\subsection{Modeling the Interaction between Microglia and Neural Stem Cells and Impact on the Brain in} Stroke (SMNR)

We formulate the following a non-linear system of ordinary differential equations to describe the interaction between microglial cells and neural stem cells and to investigate the damage to brain cells in the recovery stage after a stroke. This system uses the same equations as the SMD model, but also includes the transition from $M_{1}$ to $M_{2}$ and the behavior of neural stem cells $N_{S C}$. The system is as follows:

$$
\begin{aligned}
\frac{d M_{0}}{d t} & =\alpha-\left[R_{1}+R_{2}+\mu\right] M_{0}(t) \\
\frac{d M_{1}}{d t} & =R_{1} M_{0}(t)-\left[R_{3}+\delta D(t)+k_{1} N_{S C}(t)+\gamma_{1}\right] M_{1}(t) \\
\frac{d M_{2}}{d t} & =R_{2} M_{0}(t)+R_{3} M_{1}(t)-\left[k_{2} N_{S C}(t)+\gamma_{2}\right] M_{2}(t) \\
\frac{d N_{S C}}{d t} & =N_{S C}(t)\left[k_{2} M_{2}(t)+k_{1} M_{1}(t)-\beta_{2} C(t)-\sigma\right] \\
\frac{d D}{d t} & =D(t)\left[\delta M_{1}(t)-\beta_{1} C(t)-r_{1} M_{1}(t)-r_{2} M_{2}(t)\right] \\
\frac{d C}{d t} & =C(t)\left[\beta_{1} D(t)+\beta_{2} N_{S C}(t)-\beta_{0}\right]
\end{aligned}
$$


with initial values $M_{0}[0]=1[1], M_{1}[0]=0.02[35], M_{2}[0]=0.1514[1], N_{S C}[0]=0.85[38], D[0]=0.4$ Ref. [1], and $C[0]=0.28$ [35].

The parameters are real and positive, where $R_{3}$ denotes the rate of transition from $M_{1}$ to $M_{2}$, $k_{1}$ is the rate of interaction between neural stem cells and $M_{1}$ microglia, $k_{2}$ is the rate of interaction between neural stem cells and $M_{2}$ microglia, $\beta_{2}$ is the rate of the effect of neural stem cells on brain cells, and $\sigma$ is the death rate of neural stem cells. The other parameters are the same as in the SMD model. In the SMNR model, $M_{1}<M_{2}$ occurs during the recovery stage, where neural stem cells also start to generate and help the brain. Therefore, microglial activation is important for directing the replacement of damaged or lost cells in the brain.

\subsubsection{Equilibrium Points}

In this section, we calculate the equilibrium points of the system (11)-(16) and determine the parameter conditions for the existence of the different types of biological states. We find the steady-state solutions as follows:

$$
\frac{d M_{0}}{d t}=\frac{d M_{1}}{d t}=\frac{d M_{2}}{d t}=\frac{d N_{S C}}{d t}=\frac{d D}{d t}=\frac{d C}{d t}=0 .
$$

We classify three states of steady states:

Definition 4 (The transformation state). We define the transition cytokines from proinflammation microglia to anti-inflammation $\left(M_{1}\right.$ to $\left.M_{2}\right)$. The steady state of the form $M_{0} ; M_{1} ; M_{2}>0$ and $N_{S C}, D, C=0$ indicates the beginning of recovery stage.

Definition 5 (The interaction between microglia and neural stem cells state). We define the interaction between microglia and neural stem cells microglia in which the proinflammation phenotype $\left(M_{1}\right)$ affects negatively on neural stem cells generation and the anti-inflammation phenotype $\left(M_{2}\right)$ stimulates its generation. The steady state of the form $M_{0} ; M_{1} ; M_{2} ; N_{S C}>0$ and $C, D=0$, indicates the interaction between brain cells in the recovery stage.

Definition 6 (The impact of neural stem cells on brain cells state). We define the impact of generating neural stem cells on brain cells, the steady state of the form $M_{0} ; M_{1} ; M_{2} ; N_{S C} ; C>0$ and $D=0$ indicates the generation of neural stem cells on brain cells.

Populations of microglia cells $\left(M_{0}, M_{1}, M_{2}\right), D, N_{S C}$ and $C$ are positive or equal to zero. Thus, we obtain the equilibrium points by solving the system (11)-(16) to determine the positive equilibrium points if and only if, $M_{0}, M_{1}, M_{2}, D, C$ exemplify the positive solution. Subpopulation of microglia cells $M_{0}, M_{1}, M_{2}, N_{S C}, D$ and $C$ are positive for all $t>0$.

Proposition 2 (Nonnegative equilibriums for the SMNR model). We assume the equilibrium points for the system SMNR is nonnegative for the following conditions given by:

- $\delta>r_{1}$

- $\alpha \beta_{2}>\beta_{0} \sigma$

- $R_{2} \alpha \beta_{2}>\beta_{0} \sigma\left(R_{2}+\mu\right)$

- $k_{1} \alpha \beta_{2}>k_{1} \beta_{0} \sigma+\beta_{2} \gamma_{1} \sigma$

- $\quad\left(k_{1} \beta_{0}\left(R_{2}+\mu\right) \sigma+\beta 2\left(R_{3}+\gamma_{1}\right) x \sigma<k_{1} R_{1}\left(\alpha \beta_{2}-\beta_{0} \sigma\right)\right.$

- $k_{2} \gamma_{1}<k_{1} \gamma_{2}$

- $\quad k_{1} k_{2} \alpha+k_{2} R_{3} \sigma+k_{2} \gamma_{1} \sigma>k_{1} \gamma_{2} \sigma$

- $\quad k_{1} k_{2}\left(R_{1}+R_{2}\right) \alpha+k_{2}\left(R_{3}+\gamma_{1}\right) x \sigma<k_{1} \gamma_{2} x \sigma+v$

- $k_{1} k_{2}\left(R_{1}+R_{2}\right) \alpha+k_{2} R_{3} x \sigma+k_{1} \gamma_{2} x \sigma>k_{2} \gamma_{1} x \sigma+v$

- $k_{1} k_{2}\left(R_{1}+R_{2}\right) \alpha+v>k_{1} \gamma_{2} x \sigma+k_{2}\left(R_{3}+\gamma_{1}\right) x \sigma$. 
Then and only then there exist non-negative real steady states.

Proof. The proof is clearly found from the steady states imposing $\left(M_{0} ; M_{1} ; M_{2} ; N_{S C} ; D ; C\right)>0$. Thus the models (11)-(16) obtain three points of equilibrium in the recovery stage by using MATHEMATICA.

The first equilibrium point: the transformation state of microglia from proinflammation to anti-inflammation, $E_{t}$

Corollary 4. The equilibrium point $E_{t}$ has transformation microglia from proinflammation to anti-inflammation point recovery state from stroke given as follows:

The equilibrium point $E_{t}=\left(M_{0}, M_{1}, M_{2}, N_{S C}, D, C\right)$ exists in $\Re_{+}^{6}$, in the beginning of activation of microglia that inhibits and transfers part of $M_{1}$ phenomena to the second $M_{2}$ phenomena. Then, $C=0, D=0$, $N_{S C}=0$. This point $E_{t}$ is given by

$$
E_{t}=\left(\frac{\alpha}{x}, \frac{\left(R_{1} \alpha\right)}{\left(R_{3}+\gamma_{1}\right) x}, \frac{\alpha\left(R_{1} R_{3}+R_{2}\left(R_{3}+\gamma_{1}\right)\right)}{\left(R_{3}+\gamma 1\right) \gamma_{2} x}, 0,0,0\right),
$$

where

$$
x=R_{1}+R_{2}+\mu .
$$

The second equilibrium point: the interaction between microglia and neural stem cells on brain cells, $E_{I}$

Corollary 5. The equilibrium point $E_{I}=\left(M_{0}, M_{1}, M_{2}, N_{S C}, D, C\right)$ exists in $\Re_{+}^{6}$. At the beginning of the neural stem cells generation, the damage decreases in this state. Then, $C=0, D=0$. This point is given by

$$
\begin{aligned}
E_{I}= & \left(\frac{\alpha}{x}, \frac{1}{2 k_{1}\left(-k_{2} \gamma_{1}+k_{1} \gamma_{2}\right) x}\left[\left(R_{1}+R_{2}\right)\left(-k_{1} k_{2} \alpha-k_{2} R_{3} \sigma-k_{2} \gamma_{1} \sigma+k_{1} \gamma_{2} \sigma\right)\right.\right. \\
& \left.-\mu \sigma\left[k_{2}\left(R_{3}+\gamma_{1}\right)-k_{1} \gamma_{2}\right]+v\right], \frac{1}{2 k_{2}\left(-k_{2} \gamma_{1}+k_{1} \gamma_{2}\right) x}\left[( R _ { 1 } + R _ { 2 } ) \left(k_{1} k_{2} \alpha\right.\right. \\
& \left.\left.+k_{2} R_{3} \sigma-k_{2} \gamma_{1} \sigma+k_{1} \gamma_{2} \sigma\right)+\mu \sigma\left(k_{2}\left(R_{3}-\gamma_{1}\right)+k_{1} \gamma_{2}\right)\right]-v \\
& , \frac{1}{2 k_{1} k_{2} x \sigma}\left[( R _ { 1 } + R _ { 2 } ) \left(k_{1} k_{2} \alpha-k_{2} R_{3} \sigma-k_{2} \gamma_{1} \sigma\right.\right. \\
& \left.\left.\left.-k_{1} \gamma_{2} \sigma\right)-k_{2} \mu \sigma\left(R_{3}+\gamma_{1}\right)-k_{1} \gamma_{2} \mu \sigma+v\right), 0,0\right)
\end{aligned}
$$

where,

$$
v=\sqrt{\left(4 k_{1} k_{2} R_{1} \alpha\left(-k_{2} \gamma_{1}+k_{1} \gamma_{2}\right) x \sigma+\left(k_{1} k_{2}\left(R_{1}+R_{2}\right) \alpha+k_{2}\left(R_{3}+\gamma_{1}\right) x \sigma-k_{1} \gamma_{2} x \sigma\right)^{2}\right)} .
$$

The third equilibrium point: the effect of generating neural stem cells on brain cells, $E_{R}$

Corollary 6. The equilibrium point $E_{R}=\left(M_{0}, M_{1}, M_{2}, N_{S C}, D, C\right)$ exists in of $\Re_{+}^{6}$. When the neural stem cells are generated, the damage fades away in this state. Then, $D=0$. This point is given by:

$$
\begin{aligned}
E_{R}= & \left(\frac{\alpha}{x}, \frac{R_{1} \alpha \beta_{2}}{\epsilon_{1} x}, \frac{\alpha \beta_{2}\left(R_{1} R_{3} \beta_{2}+R_{2} \epsilon_{1}\right)}{\epsilon_{1} \epsilon_{2} x}, \frac{\beta_{0}}{\beta_{2}}, 0\right. \\
& ,\left(\frac{\beta_{2} \gamma_{2}\left(-k_{1} \beta_{0}\left(R_{2}+\mu\right) \sigma-\beta_{2}\left(R_{3}+\gamma_{1}\right) x \sigma+k_{1} R_{1} \epsilon_{3}\right)+k_{2} \epsilon_{7}}{\beta_{2} \epsilon_{1} \epsilon_{2} x}\right),
\end{aligned}
$$


where,

$$
\begin{aligned}
& \epsilon_{1}=\left(R_{3} \beta_{2}+k_{1} \beta_{0}+\beta_{2} \gamma_{1}\right), \epsilon_{2}=k_{2} \beta_{0}+\beta_{2} \gamma_{2}, \\
& \epsilon_{3}=\left(\alpha \beta_{2}-\beta_{0} \sigma\right), \epsilon_{4}=\left(-k_{1} \alpha \beta_{2}+k_{1} \beta_{0} \sigma+\beta_{2} \gamma_{1} \sigma\right), \\
& \epsilon_{5}=R_{3} \beta_{2}+k_{1} \beta_{0}+\beta_{2} \gamma_{1}, \epsilon_{6}=\left(R_{2} \alpha \beta_{2}-R_{2} \beta_{0} \sigma-\beta_{0} \mu \sigma\right), \\
& \epsilon_{7}=R_{1} R_{3} \beta_{2} \epsilon_{3}-R_{1} \beta_{0} \epsilon_{4}+\epsilon_{5} \epsilon_{6} .
\end{aligned}
$$

\subsubsection{Stability of Equilibrium Points for SMNR Model}

We study the stability of the equilibrium points for the second model SMNR by using Definitions 4-6. The first step requires linearization of the system equations that describes the interaction between microglia $M_{1}, M_{2}$, neural stem cells $N_{S C}$, the damage $D$ in this stage and the impact it has on the brain cells $C$. The $(6 \times 6)$ Jacobian matrix of the system $(11)-(16)$ is given by:

$$
J_{2}=\left[\begin{array}{cccccc}
j_{11} & 0 & 0 & 0 & 0 & 0 \\
R_{1} & j_{22} & 0 & -k_{1} M_{1} & -M_{1} \delta & 0 \\
R_{2} & R_{3} & j_{33} & -k_{2} M_{2} & 0 & 0 \\
0 & k_{1} N_{S C} & k_{2} N_{S C} & j_{54} & 0 & -N_{S C} \beta_{2} \\
0 & D\left(-r_{1}+\delta\right) & -D r_{2} & 0 & j 55 & -D \beta_{1} \\
0 & 0 & 0 & c \beta_{2} & c \beta_{1} & j_{66}
\end{array}\right],
$$

where,

$$
\begin{aligned}
& j_{11}=-x, x=R_{1}+R_{2}+\mu, j_{22}=-R_{3}-k_{1} N_{S C}-\gamma_{1}-D \delta, \\
& j_{33}=-k_{2} N_{S C}-\gamma_{2}, j_{54}=k_{1} M_{1}+k_{2} M_{2}-C \beta_{2}-\sigma, \\
& j_{55}=-M_{2} r_{2}-C \beta_{1}+M_{1}\left(-r_{1}+\delta\right), \quad j_{66}=D \beta_{1}+N_{S C} \beta_{2}-\beta_{0} .
\end{aligned}
$$

1. Stability analysis of equilibrium point, $E_{t}$ :

Theorem 4. Suppose that the function $f: \Gamma \rightarrow \Re_{+}^{6}$ where $\Gamma$ is a domain in $\Re_{+}^{6}$, and suppose that $E_{t}=\left(M_{0}, M_{1}, M_{2}, 0,0,0\right) \in \Gamma$ is an equilibrium point at which at least one eigenvalue of the Jacobian matrix has a positive real part. Then, $E_{t}$ is an unstable equilibrium point of $f$.

Proof. The Jacobian $J_{2}$ corresponding to the equilibrium point $E_{t}$ is given by

$$
J\left[E_{t}\right]=\left[\begin{array}{cccccc}
-x & 0 & 0 & 0 & 0 & 0 \\
R_{1} & -R_{3}-\gamma_{1} & 0 & d_{24} & d_{25} & 0 \\
R_{2} & R_{3} & -\gamma_{2} & d_{34} & 0 & 0 \\
0 & 0 & 0 & d_{44} & 0 & 0 \\
0 & 0 & 0 & 0 & d_{55} & 0 \\
0 & 0 & 0 & 0 & 0 & -\beta_{0}
\end{array}\right]
$$

where,

$$
\begin{aligned}
& d_{24}=-\frac{k_{1} R_{1} \alpha}{\left(R_{3}+\gamma_{1}\right) x}, d_{25}=-\frac{R_{1} \alpha \delta}{\left(R 3+\gamma_{1}\right) x}, \\
& d_{34}=-\frac{k_{2} \alpha\left(R_{1} R_{3}+R_{2}\left(R_{3}+\gamma_{1}\right)\right)}{\left(R_{3}+\gamma_{1}\right) \gamma_{2} x}, d_{44}=\frac{k_{1} R_{1} \alpha}{\left(R_{3}+\gamma_{1}\right) x}+\frac{k_{2} \alpha\left(R_{1} R_{3}+R_{2}\left(R_{3}+\gamma_{1}\right)\right)}{\left(R_{3}+\gamma_{1}\right) \gamma_{2} x}-\sigma, \\
& d_{55}=-\frac{r_{2} \alpha\left(R_{1} R_{3}+R_{2}\left(R_{3}+\gamma_{1}\right)\right)}{\left(R_{3}+\gamma_{1}\right) \gamma_{2} x}+\frac{R_{1} \alpha\left(-r_{1}+\delta\right)}{\left(R_{3}+\gamma_{1}\right) x} .
\end{aligned}
$$


From the Jacobian matrix $J\left[E_{t}\right]$, the characteristic equation is given by

$$
\left(\beta_{0}+\lambda\right)\left(R_{3}+\gamma_{1}+\lambda\right)\left(\gamma_{2}+\lambda\right)(x+\lambda)\left(-\lambda+t_{55}\right)\left(-\lambda+t_{44}\right)=0 .
$$

Then, the eigenvalues corresponding to $E_{t}$ are given by

$$
\begin{aligned}
& \lambda_{1}=-\beta_{0}<0, \lambda_{2}=-R_{3}-\gamma_{1}<0, \lambda_{3}=-x<0, \lambda_{4}=-\gamma_{2}<0, \\
& \lambda_{5}=\frac{k_{1} R_{1} \alpha}{\left(R_{3}+\gamma_{1}\right) x}+\frac{k_{2} \alpha\left(R_{1} R_{3}+R_{2}\left(R_{3}+\gamma_{1}\right)\right)}{\left(R_{3}+\gamma_{1} \gamma_{2}\right) x}-\sigma>0, \\
& \lambda_{6}=-\frac{r_{2} \alpha\left(R_{1} R_{3}+R_{2}\left(R_{3}+\gamma_{1}\right)\right)}{\left(R_{3}-\gamma_{1} \gamma_{2}\right) x}+\frac{\left(R_{1} \alpha\left(r_{1}-\delta\right)\right)}{\left(R_{3}+\gamma_{1}\right) x}<0 .
\end{aligned}
$$

One of the eigenvalues, $\lambda_{5}>0$, then $E_{t}$ is an unstable point.

2. Stability analysis of equilibrium point, $E_{I}$ :

Theorem 5. Suppose that the function $f: \Gamma \rightarrow \Re_{+}^{6}$ where $\Gamma$ is a domain in $\Re_{+}^{6}$, and suppose that $E_{I}=$ $\left(M_{0}, M_{1}, M_{2}, N_{S C}, 0,0\right) \in \Gamma$ is an equilibrium point at which at least one eigenvalue of the Jacobian matrix has a positive real part and $N_{S C}>0$. Then, $E_{I}$ is an unstable equilibrium point of $f$.

Proof. The Jacobian matrix $J_{2}$ corresponding to the equilibrium point $E_{I}$ is given by:

$$
J\left[E_{I}\right]=\left[\begin{array}{cccccc}
-x & 0 & 0 & 0 & 0 & 0 \\
R_{1} & e_{22} & 0 & e_{24} & e_{25} & 0 \\
R_{2} & R_{3} & e_{33} & e_{34} & 0 & 0 \\
0 & e_{42} & e_{43} & 0 & 0 & e_{46} \\
0 & 0 & 0 & 0 & e_{55} & 0 \\
0 & 0 & 0 & 0 & 0 & e_{66}
\end{array}\right],
$$

where,

$$
\begin{aligned}
e_{22}= & -\frac{\Lambda_{1}+v}{2 k_{2} x \sigma}<0, e_{42}=\frac{\Lambda_{2}}{2 k_{2} x \sigma}>0, e_{33}=-\frac{\Lambda_{2}}{2 k_{1} x \sigma}<0, e_{43}=\frac{\Lambda_{2}}{2 k_{1} x \sigma}>0, \\
e_{24}= & \frac{-\Lambda_{1}+v}{2 q x}<0, e_{34}=\frac{\Lambda_{3}}{2 q x}<0, e_{25}=-\frac{\delta \Lambda_{4}}{2 k_{1} q x}<0, \\
e_{46}= & \frac{\Lambda_{7}}{2 k_{1} k_{2} x \sigma}<0, e_{55}=-\frac{\Lambda_{5}}{2 k_{1} k_{2} q x}>0, e_{66}=\frac{\Lambda_{6}}{2 k_{1} k_{2} x \sigma}>0, \\
v= & \sqrt{4 k_{1} k_{2} R_{1} \alpha\left(-k_{2} \gamma_{1}+k_{1} \gamma_{2}\right) x \sigma+\left(k_{1} k_{2}\left(R_{1}+R_{2}\right) \alpha+k_{2}\left(R_{3}+\gamma_{1}\right) x \sigma-k_{1} \gamma_{2} x \sigma\right)^{2}}>0, \\
\Psi= & x\left(k_{2}\left(R_{3}+\gamma_{1}\right)+k_{1} \gamma_{2}\right) \sigma>0, \Lambda_{1}=k_{1} k_{2}\left(R_{1}+R_{2}\right) \alpha+k_{2} x\left(R_{3}+\gamma_{1}\right) \sigma-k_{1} x \gamma_{2} \sigma>0, \\
\Lambda_{2}= & v-\Psi+k_{1} k_{2}\left(R_{1}+R_{2}\right) \alpha>0 \\
\Lambda_{3}= & -v+k_{1} k_{2}\left(R_{1}+R_{2}\right) \alpha+k_{2} x\left(R_{3}-\gamma_{1}\right) \sigma+k_{1} x \gamma_{2} \sigma>0, \\
\Lambda_{4}= & -v+k_{1} k_{2}\left(R_{1}+R_{2}\right) \alpha+\left(k_{2}\left(R_{3}+\gamma_{1}\right)-k_{1} \gamma_{2}\right) x \sigma<0, \Lambda_{5}=k_{2}^{2}\left(r_{1}-\delta\right)\left(k_{1}\left(R_{1}+R_{2}\right) \alpha\right. \\
& \left.+x\left(R_{3}+\gamma_{1}\right) \sigma\right)+k_{1} r_{2}\left(v-k_{1} x \gamma_{2} \sigma\right)+k_{2}\left(-k_{1} r_{2}\left(k_{1}\left(R_{1}+R_{2}\right) \alpha+x\left(R_{3}-\gamma_{1}\right) \sigma\right)\right. \\
& \left.-\left(r_{1}-\delta\right)\left(v+k_{1} x \gamma_{2} \sigma\right)\right)>0, \\
\Lambda_{6}= & v \beta_{2}+k_{1} k_{2}\left(R_{1}+R_{2}\right) \alpha \beta_{2}-x\left(k_{2} \beta_{2}\left(R_{3}+\gamma_{1}\right)+k_{1}\left(2 k_{2} \beta_{0}+\beta_{2} \gamma_{2}\right)\right) \sigma>0, \\
\Lambda_{7}= & \beta_{2}\left(-v-k_{1} k_{2}\left(R_{1}+R_{2}\right) \alpha+k_{2} x\left(R_{3}+\gamma_{1}\right) \sigma+k_{1} x \gamma_{2} \sigma\right)<0, \\
q= & k_{2} \gamma_{1}-k_{1} \gamma_{2}<0, x=R_{1}+R_{2}+\mu>0 .
\end{aligned}
$$


From the Jacobian $J\left[E_{I}\right]$, the characteristic equation is given by

$$
\left(e_{66}-\lambda\right)(\lambda+x)\left(e_{55}-\lambda\right)\left[\lambda^{3}+T_{2} \lambda^{2}+T_{1} \lambda+T_{0}\right]=0,
$$

where,

$$
\begin{aligned}
& T_{3}=-8 k_{1}^{3} k_{2}^{2} q^{2} x^{4} \sigma^{3}, T_{2}=-4 k_{1}^{2} k_{2} q^{2}\left(k_{1}\left(v+\Lambda_{1}\right)+k_{2} \Lambda_{2}\right) x^{3} \sigma^{2}, \\
& T_{1}=2 k_{1}^{2} k_{2} q x^{2} \Lambda_{2} \sigma\left(-q\left(v+\Lambda_{1}\right)+\Lambda_{2}\left(k_{1}\left(v-\Lambda_{1}\right)+k_{2} \Lambda_{3}\right) \sigma\right), \\
& T_{0}=k_{1}^{2} k_{2} q \Lambda_{2} x \sigma\left(\Lambda_{1}\left(-\Lambda_{2}+\Lambda_{3}-2 k_{2} R_{3} x \sigma\right)+v\left(\Lambda_{2}+\Lambda_{3}+2 k_{2} R_{3} x \sigma\right)\right) .
\end{aligned}
$$

In Equation (21), the eigenvalue $\lambda=e_{66}$ is distinctly positive, by Proposition 2 . Thus, $J\left(E_{I}\right)$ has at least one positive root. Thus, the equilibrium point $E_{I}$ is unstable [44].

3. Stability analysis of equilibrium point, $E_{R}$ :

Theorem 6. Suppose that the function $f: \Gamma \rightarrow \Re_{+}^{6}$ where $\Gamma$ is a domain in $\Re_{+}^{6}$, and suppose that $E_{R}=\left(M_{0}, M_{1}, M_{2}, N_{S C}, 0, C\right) \in \Gamma$ is an equilibrium point at which at least one eigenvalue of the Jacobian matrix has a positive real part and $N_{S C}>0, C>0$. Then, $E_{R}$ is an unstable equilibrium point of $f$.

Proof. We now study the stability of the equilibrium point $E_{R}$, calculated as: The Jacobian matrix $J_{2}$ estimated at $E_{R}$ is

$$
J\left[E_{R}\right]=\left[\begin{array}{cccccc}
-x & 0 & 0 & 0 & 0 & 0 \\
R_{1} & f_{22} & 0 & f_{24} & f_{25} & 0 \\
R_{2} & R_{3} & f_{33} & f_{34} & 0 & 0 \\
0 & \frac{k_{1} \beta_{0}}{\beta_{2}} & \frac{k_{2} \beta_{0}}{\beta_{2}} & f_{44} & 0 & -\beta_{0} \\
0 & f_{52} & f_{53} & 0 & f_{55} & f_{56} \\
0 & 0 & 0 & 0 & 0 & f_{66}
\end{array}\right],
$$

where

$$
\begin{aligned}
f_{22}= & -R_{3}-\frac{k_{1} \beta_{0}}{\beta_{2}}-\gamma_{1}-\frac{\delta \rho_{1}}{\beta_{2} v_{1} v_{2} x}<0, f_{24}=-\frac{k_{1} R_{1} \alpha \beta_{2}}{v_{1} x}<0, f_{25}=-\frac{\left(R_{1} \alpha \beta_{2} \delta\right)}{v_{1} x}<0, \\
f_{55}= & -\frac{\left(r_{2} \alpha \beta_{2}\right) \rho_{2}}{v_{1} v_{2} x}+\frac{R_{1} \alpha \beta_{2}\left(-r_{1}+\delta\right)}{v_{1} x}>0, f_{33}=-\frac{k_{2} \beta_{0}}{\beta_{2}}-\gamma_{2}, f 34=-\frac{k_{2} \alpha \beta_{2} \rho_{2}}{v_{1} v_{2} x}>0, \\
f_{43}= & \frac{k_{2} \beta_{0}}{\beta_{2}}, f_{44}=\frac{k_{1} R_{1} \alpha \beta_{2}}{v_{1} x}+\frac{k_{2} \alpha \beta_{2} \rho_{2}}{v_{1} v_{2} x}-\sigma>0, f_{42}=\frac{k_{1} \beta_{0}}{\beta_{2}}, f_{53}=-\frac{r_{2} \rho_{1}}{\beta_{2} v_{1} v_{2} x}<0, \\
f_{56}= & -\frac{\beta_{1} \rho_{1}}{\beta_{2} v_{1} v_{2} x}<0, f_{52}=\frac{\left(-r_{1}+\delta\right) \rho_{1}}{\beta_{2} v_{1} v_{2} x}<0, f_{66}=\frac{\beta_{1} \rho_{1}}{\beta_{2} v_{1} v_{2} x}>0, \\
\rho_{1}= & \left(\beta_{2} \gamma_{2}\left(-k_{1} \beta_{0}\left(R_{2}+\mu\right) \sigma-\beta_{2}\left(R_{3}+\gamma_{1}\right) x \sigma+k_{1} R_{1}\left(\alpha \beta_{2}-\beta_{0} \sigma\right)\right),\right. \\
& +k_{2}\left(R_{1} R_{3} \beta_{2}\left(\alpha \beta_{2}-\beta_{0} \sigma\right)\right)-R_{1} \beta_{0}\left(-k_{1} \alpha \beta_{2}+k_{1} \beta_{0} \sigma+\beta_{2} \gamma_{1} \sigma\right) \\
& \left.\left.+\left(R_{3} \beta_{2}+k_{1} \beta_{0}+\beta_{2} \gamma_{1}\right)\left(R_{2} \alpha \beta_{2}-R_{2} \beta_{0} \sigma-\beta_{0} \mu \sigma\right)\right)\right) \\
\rho_{2}= & \left(R_{1} R_{3} \beta_{2}+R_{2}\left(R_{3} \beta_{2}+k_{1} \beta_{0}+\beta_{2} \gamma_{1}\right)\right)>0, \\
v_{1}= & \left(R_{3} \beta_{2}+k_{1} \beta_{0}+\beta_{2} \gamma_{1}\right)>0, v_{2}=\left(k_{2} \beta_{0}+\beta_{2} \gamma_{2}\right)>0 .
\end{aligned}
$$

From the Jacobian $J\left[E_{R}\right]$, the characteristic equation is given by

$$
\begin{aligned}
\left(f_{66}-\lambda\right)(\lambda & +x)\left(f_{25} f_{34}\left(-f_{53} k_{1}+f_{52} k_{2}\right) \beta_{0}+f_{25} \beta_{2}\left(f_{44}-\lambda\right)\left(-f_{33} f_{52}+f_{53} R_{3}+f_{52} \lambda\right)+\left(f_{55}-\lambda\right)\right. \\
& \left.\times\left(\left(-f_{34} k_{2} \beta_{0}+\beta_{2}\left(f_{33}-\lambda\right)\left(f_{44}-\lambda\right)\right)\left(f_{22}-\lambda\right)+f_{24} \beta_{0}\left(-f_{33} k_{1}+k_{2} R_{3}+k_{1} \lambda\right)\right)\right)=0 .
\end{aligned}
$$


In Equation (22), the eigenvalue $\lambda=f_{66}$ is distinctly positive, by Proposition 2 . Thus, $J\left(E_{R}\right)$ has at least one positive root and, so, the equilibrium point $E_{R}$ is unstable [44].

Remark 2. The effects of microglia and neural stem cells on brain cells in the recovery stage after a stroke, according to analysis of the dynamic system of SMNR, can be deduced as follows:

- As a result of Theorem 4 and Definition 4, the neural stem cells, Nsc, can invade the SMNR system if $\lambda_{5}>0$.

- As a result of Theorem 5 and Definition 5, this means that the neural stem cells, Nsc > 0, can invade $C$ and $D$.

- As a result of Theorem 6 and Definition 6, this means that the neural stem cells, Nsc $>0$, can eliminate the damage $D$.

- $\quad$ The SMNR model is unstable, given that the model uses mature neural stem cells, where the neural stem cells can help the brain to inhibit inflammation during a stroke; however, it is not always enough to substitute all dead cells with new neural stem cells [3,27].

\section{Numerical Experiments}

The aim of this section is to study the parameters of the systems (1)-(5) and (11)-(16), in order to determine those that affect the behavior of the modified models, by using numerical simulations. One of the main problems in modeling and simulating the interactions between microglia and endogenous neural stem cells is that few parameter values are known. Therefore, we listed the parameters which could be determined from the stroke model of [1]. The other parameters were determined from experimental data of other brain injuries which involve similar biological processes. Furthermore, we obtained some parameter values by simulations using the Mathematica software (11.2, Wolfram, Champaign, IL, USA) with the command NDSolve, in order to study the influence of the interactions during the stroke and the possible therapeutic value of neural stem cells. Tables 1 and 2 show the reference sets of parameter values, along with the corresponding simulation results.

Table 1. Parameters values for the stroke-microglia-damage (SMD) model.

\begin{tabular}{lccc}
\hline The SMD Model & & & \\
\hline Parameters & Values & Meaning & Sources \\
\hline$\alpha$ & 0.38 & Source of resting microglia & {$[1]$} \\
$R_{1}$ & 0.12 & Rate the activation of resting microglia into $M_{1}$ & {$[1]$} \\
$R_{2}$ & 0.017 & Rate the activation of resting microglia into $M_{2}$ & {$[35]$} \\
$\delta$ & 0.2854 & Rate of the damage produced by $M_{1}$ & {$[1]$} \\
$\beta_{1}$ & 0.1 & Rate of effect the damage on $C(t)$ & {$[1]$} \\
$\beta_{0}$ & 0.05 & Rate of dying $C$ through stroke & {$[1]$} \\
$\mu$ & 0.003 & Death rate of $M_{0}$ & simulation \\
$\gamma_{1}$ & 0.05 & Death rate of $M_{1}$ & simulation \\
$\gamma_{2}$ & 0.06 & Death rate of $M_{2}$ & simulation \\
$r_{1}$ & 0.05 & Damage clearance by $M_{1}$ & {$[1]$} \\
$r_{2}$ & 0.0125 & Damage clearance by $M_{2}$ & \\
\hline
\end{tabular}

The numerical simulations in Tables 1 and 2 appeared in the SMD and the SMNR models. The microglial cells at stroke onset had high proinflammatory activation (i.e., high level of $M_{1}$ ), which caused an increase of damage in the region, affecting brain cells as well as neural stem cells during the $72 \mathrm{~h}$ period. On the other hand, when the rate of the anti-inflammatory phenotype microglia $\left(M_{2}\right)$ became higher than that of the $M_{1}$, the brain was assisted in recovery and the neural stem cells were stimulated to generate new cells to compensate for lost brain cells. These dynamics started approximately on the third day after stroke onset. The parameters of the SMD model, in the simulation, were fixed as $\mu=0.003, \gamma_{1}=0.05$, and $\gamma_{2}=0.06$. The parameters of the SMNR model, in the simulation, were fixed as $R_{3}=0.11, K_{1}=0.75, K_{2}=0.91, \beta_{2}=0.2$, and $\mu=0.053$. In the 
SMD model, $M_{1}>M_{2}$ and we set $R_{1}=0.12$ [1] and $R_{2}=0.017$ [35]. In the SMNR model, $M_{2}>M_{1}$, the source of resting microglia was $\alpha=0.38$, and the rate of the anti-inflammatory cytokines $\left(R_{2}\right)$ was greater than that of the proinflammatory cytokines $\left(R_{1}\right)$ in the recovery stage. Accordingly, we set $R_{1}=0.12$ [1] and estimated $R_{2}=0.26$. The dynamics of the systems were given by solving the systems numerically and then plotting the time series of the solutions of the system (1)-(5) and (11)-(16) for the parameters, which were solved using the fourth-order Runge-Kutta (RK4) method to obtain more stable and convergent solutions. The simulations of SMD and SMNR were carried out for the time spans of three days (72 h) and thirty days, respectively with a step size of $10^{-4}$ in the RK4 method. The SMD model described the inflammatory process where activation of the microglia cells peaks around three days $(72 \mathrm{~h})$ from stroke onset [1,2]. Neural stem cells increase the proliferation and the migration from the subventricular zone to the brain in thirty days and peaking around seven days after stroke $[3,27]$. Therefore, the SMNR model studied thirty days after stroke to investigate the interactions between microglia and neurogenesis after stroke. The accuracy of solutions by using the RK4 method of the SMD model and the best numerical solution of the model was obtained at 38 steps. While the accuracy of solutions by using the RK4 method of the SMNR model and the best numerical solution of the model was obtained at 35 steps. The reliability and accuracy of the proposed numerical method can be seen from the residual error which is shown in Figures 1 and 2. Figure 1 shows the residual error for the SMD model at the number of steps and the residual error at the time. While Figure 2 shows the residual error for the SMNR model at the number of steps and the residual errors at the time. By comparing the results in our study of microglial activation and the damage during the first three days after stroke onset and the recovery stage within the first thirty days after stroke onset with the effects of neural stem cells and those of the study in [40], we found that the studies agreed, in that the extended proinflammatory activation of microglia may inhibit neurogenesis and contribute to additional neuronal loss. Hence, the behavioural responses of microglia can lead to an increase in damage in the brain or its capability to recover. In this study, we focused on the importance and contribution of neural stem cells in transitioning to the recovery stage, as well as the interaction between microglia and neural stem cells. The generation of neural stem cells was considered to lead to a decrease in damage to the brain, compared to their absence. The behaviors of the solutions of the SMD and SMNR models are shown in Figure 3. It can be seen, in the SMD model, that the population of microglial cells $M_{1}$ had a shallow increase, asides from shifting to a steep curve at approximately 15-30 h, and peaked at around $72 \mathrm{~h}$. On the other hand, we can observe that $M_{2}$ had a low level after stroke onset and, after three days, reached a stable level. The population of $M_{1}$ began to shift into the steep curve (the second stage) with an increase of $M_{2}$. Furthermore, the damage increased sharply in the initial stage and decreased in the second stage. On the other hand, in the SMNR model, the response of the neural stem cells began after the initial stage, helping the brain cells to repair damaged cells and lost cells, depending on whether the number of living cells was greater than that of the dead cells. As depicted in Figure 3, the curve of neural stem cells increased and then decreased during the 15 days following stroke onset; this can be explained by the ability of neural stem cells to inhibit inflammation, as has been studied in [3], where the survival of neural stem cells seemed to be prevented, with up to $80 \%$ of the new neurons dying within two weeks after their generation in vivo. Furthermore, the curve of neural stem cells in Figure 3 reached its peak during the first week, in agreement with studies in rats and mice, where the increase in proliferation of neural stem cells peaked at around 7 days after ischemic injury [27]. 
Table 2. Parameters values for the stroke-microglia-neural stem cells-recovery (SMNR) model.

\begin{tabular}{|c|c|c|c|}
\hline \multicolumn{4}{|c|}{ The SMNR Model } \\
\hline Parameters & Values & Biological Meaning & Sources \\
\hline$\alpha$ & 0.38 & Source of resting microglia & [1] \\
\hline$R_{1}$ & 0.12 & Rate the activation of resting microglia into $M_{1}$ & [1] \\
\hline$R_{2}$ & 0.26 & Rate the activation of resting microglia into $\mathrm{M}_{2}$ & estimated \\
\hline$R_{3}$ & 0.11 & The transition rate of $M_{1} \rightarrow M_{2}$ & simulation \\
\hline$K_{1}$ & 0.91 & Rate of interaction between $M_{1}$ and $N_{S C}$ & simulation \\
\hline$K_{2}$ & 0.75 & Rate of interaction between $M_{2}$ and $N_{S C}$ & simulation \\
\hline$\delta$ & 0.2854 & Rate of the damage produced by $M_{1}$ & [1] \\
\hline$\beta_{1}$ & 0.1 & Rate of effect the damage on $C(t)$ & [1] \\
\hline$\beta_{2}$ & 0.2 & Rate of effect $N_{S C}$ on $C(t)$ & simulation \\
\hline$\beta_{0}$ & 0.05 & Rate of dying $C$ through stroke & [1] \\
\hline$\mu$ & 0.053 & Death rate of $M_{0}$ & simulation \\
\hline$\gamma_{1}$ & 0.015 & Death rate of $M_{1}$ & [35] \\
\hline$\gamma_{2}$ & 0.015 & Death rate of $M_{2}$ & [35] \\
\hline$\sigma$ & 0.015 & Death rate of $N_{S C}$ & [38] \\
\hline$r_{1}$ & 0.05 & Damage clearance by $M_{1}$ & [1] \\
\hline$r_{2}$ & 0.0125 & Damage clearance by $M_{2}$ & [1] \\
\hline
\end{tabular}
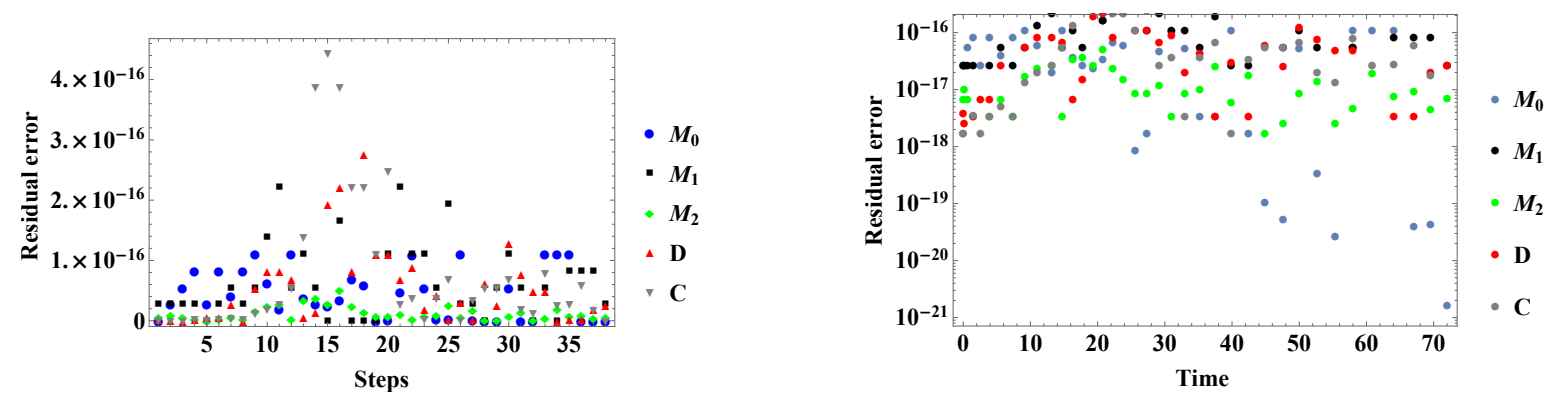

Figure 1. Residual error for the step and time of the numerical method in the SMD model.
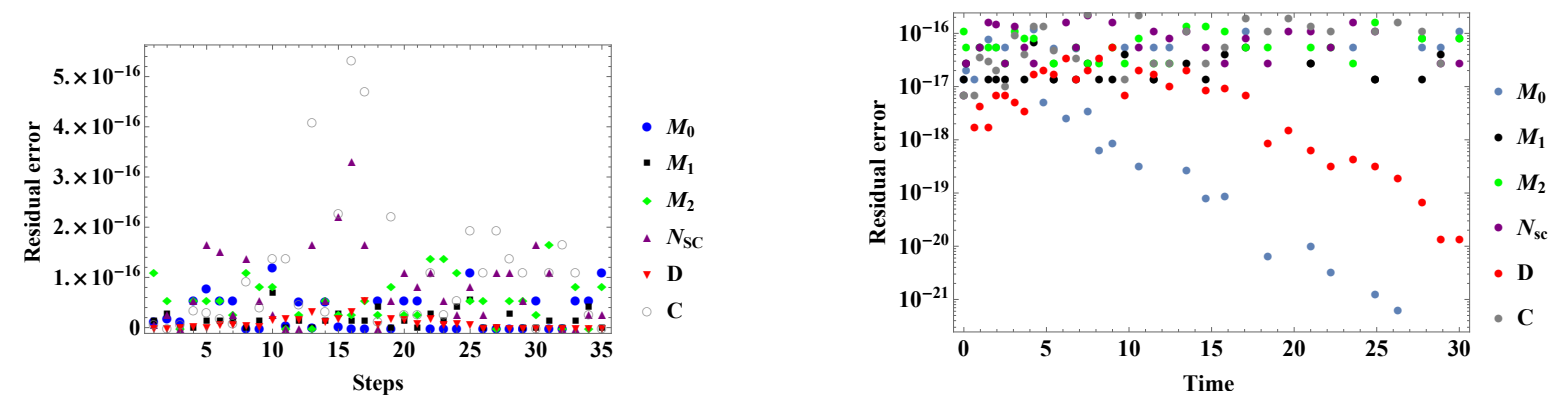

Figure 2. Residual error for the step and time of the numerical method in the SMNR model.
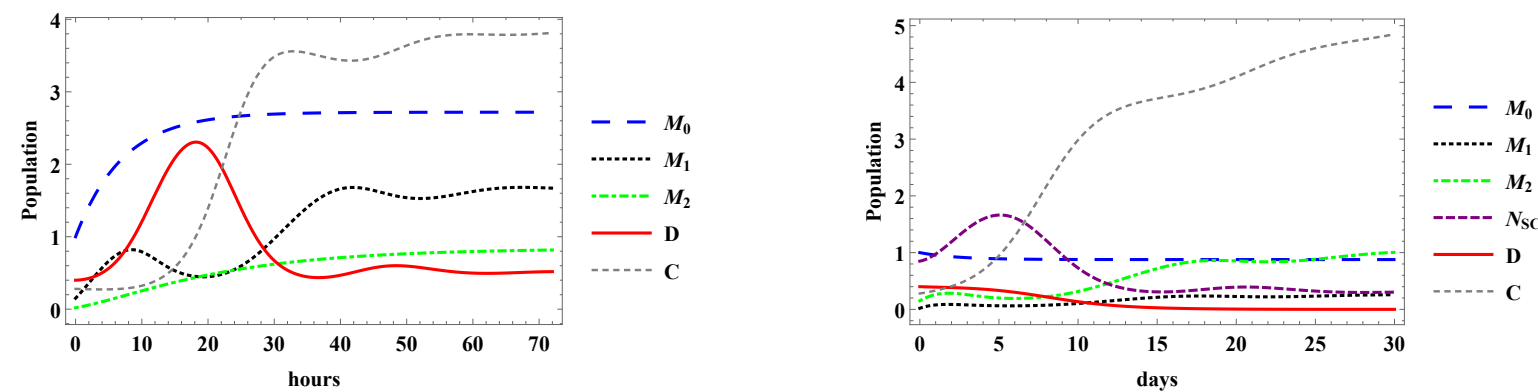

Figure 3. The behaviour of the SMD model within $72 \mathrm{~h}$ from stroke onset and the SMNR model within 30 days after stroke onset. 


\section{Conclusions}

We modified two models-SMD ((1)-(5)) and SMNR ((11)-(16))-based on the model of Leah et al. [40], in order to study the impact of the microglial activation stage and the interaction between microglia and neural stem cells during a stroke. The dynamic models of the effects of microglia and the interaction between neural stem cells and microglia in stroke over time were studied both analytically and numerically. From the results of the analysis and simulation of both models, two states of microglial cells $\left(M_{1}\right.$ to $\left.M_{2}\right)$ emerged from the resting state. In the first disease stage, the activation of microglial cells went from resting-state microglia to either of the states $M_{1}$ or $M_{2}$. The results of this interaction led to increased damage in the brain after stroke onset when $M_{2}$ was very low, there was no shift from $M_{1}$ to $M_{2}$, and no generation of neural stem cells occurred in the first $72 \mathrm{~h}$ after the stroke. Subsequently, in the recovery stage, the rate of $M_{1}$ decreased and the rate of $M_{2}$ increased and the neural stem cells began to generate. Subsequent to that, the rate of damage decreased. The instability of the SMNR model can be explained: in the nervous system, the number of endogenous neural stem cells is very low under normal physiological conditions, showing a very limited capacity for cell replacement under normal physiological conditions [20,21]. Understanding the biology in vivo of neural stem cells could lead to new therapeutic strategies for brain repair by endogenous neural stem cells [20,21], encouraging inflammatory inhibition. In conclusion, our modified models can lead to lead to an understanding of the effectiveness risks of the inflammatory responses associated with strokes and their positive and negative effects on the brain in stroke patients, as well as the general dynamics of microglial effects on neural stem cells, both during stroke and in the recovery stage. In the future, we will expand this work to study the mechanisms that improve, stimulate, and generate the neural stem cells in the early stage, and where that information could contribute to understanding the effects of therapeutic strategies. Additionally, it could be interesting to incorporate the dynamics of anti-inflammatory and proinflammatory cytokines from microglia and the cytokines of endogenous neural stem cells into the SMNR model, in order to describe the interaction processes of the different types of cytokines in ischemic stroke.

Author Contributions: Conceptualization, A.J.A.; funding acquisition, A.S.R.; methodology, A.J.A.; Project administration, A.S.R.; supervision, A.S.R. and I.H.; validation, A.S.R.; writing-original draft, A.J.A.; writing-review and editing, I.H. All authors have read and agreed to the published version of the manuscript.

Funding: This research is funded by a grant from Universiti Kebangsaan Malaysia.

Acknowledgments: This study was supported by a research grant from the Universiti Kebangsaan Malaysia with code GUP-2017-112.

Conflicts of Interest: The authors declare no conflict of interest.

\section{References}

1. Di Russo, C. A mathematical model of inflammation during ischemic stroke. Math. Model. Med. 2010, 30, 15-33. [CrossRef]

2. Guruswamy, R.; ElAli, A. Complex roles of microglial cells in ischemic stroke pathobiology: New insights and future directions. Int. J. Mol. Sci. 2017, 18, 496. [CrossRef]

3. Tobin, M.K.; Bonds, J.A.; Minshall, R.D.; Pelligrino, D.A.; Testai, F.D.; Lazarov, O. Neurogenesis and inflammation after ischemic stroke: What is known and where we go from here. J. Cereb. Blood Flow Metab. 2014, 10, 1573-1584. [CrossRef] [PubMed]

4. Radak, D.; Katsiki, N.; Resanovic, I.; Jovanovic, A.; Sudar-Milovanovic, E., Zafirovic, S.; Mousad, S.A.; Isenovic, E.R. Apoptosis and acute brain ischemia in ischemic stroke. Curr. Vasc. Pharmacol. 2017, 15, 115-122. [CrossRef] [PubMed]

5. Anttila, J.E.; Whitaker, K.W.; Wires, E.S.; Harvey, B.K.; Airavaara, M. Role of microglia in ischemic focal stroke and recovery: Focus on toll-like receptors. Neuro-Psychopharmacol. Biol. Psychiatry 2016, 79, 3-14. [CrossRef] [PubMed] 
6. Stonesifer, C.; Corey, S.; Ghanekar, S.; Diamandis, Z.; Acosta, S.A.; Borlongan, C.V. Stem cell therapy for abrogating stroke-induced neuroinflammation and relevant secondary cell death mechanisms. Prog. Neurobiol. Prog. Neurobiol. 2017, 158, 94-131. [CrossRef]

7. Banjara, M.; Ghosh, C. Sterile neuroinflammation and strategies for therapeutic intervention. Int. J. Inflamm. 2017, 10, 8385961. [CrossRef]

8. Rajab, N.F.; Musa, S.M.; Ahmad Munawar, M.; Leong, L.M.; Heng, K.Y.; Ibrahim, F.W.; Chan, K.M. Antineuroinflammatory effects of hibiscus sabdariffa Linn.(Roselle) on Lipopolysaccharides-induced Microglia and Neuroblastoma Cells. Malays. J. Health Sci. 2016, 14, 111-117.

9. Rune, L.; Martin, W.; Daniel, G.; Christina, F.; Lasse, D.; Ishar, D.; Bente, F. Microglial cell population dynamics in the injured adult central nervous system. Brain Res. Rev. 2005, 48, 196-206.

10. Galloway, D.A.; Phillips, A.E.; Owen, D.R.; Moore, C.S. Phagocytosis in the Brain: Homeostasis and Disease. Front. Immunol. 2019, 10, 790. [CrossRef]

11. Pham-Huy, L.A.; He, H.; Pham-Huy, C. Free radicals, antioxidants in disease and health. Int. J. Biomed. Sci. IJBS 2008, 4, 89-96. [PubMed]

12. Di Meo, S.; Reed, T.T.; Venditti, P.; Victor, V.M. Role of ROS and RNS Sources in Physiological and Pathological Conditions. Oxid. Med. Cell. Longev. 2016, 2016, 1245049. [CrossRef]

13. Fresta, C.G.; Chakraborty, A.; Wijesinghe, M.B.; Amorini, A.M.; Lazzarino, G.; Lazzarino, G.; Tavazzi, B.; Lunte, S.M.; Caraci, F.; Dhar, G.; et al. Non-toxic engineered carbon nanodiamond concentrations induce oxidative/nitrosative stress, imbalance of energy metabolism, and mitochondrial dysfunction in microglial and alveolar basal epithelial cells. Cell Death Dis. 2018, 9, 245. [CrossRef] [PubMed]

14. Vay, S.U.; Flitsch, L.J.; Rabenstein, M.; Rogall, R.; Blaschke, S.; Kleinhaus, J.; Bach, A.; Fink, G.R.; Schroeter, M.; Rueger, A.M. The plasticity of primary microglia and their multifaceted effects on endogenous neural stem cells in vitro and in vivo. J. Neuroinflamm. 2018, 15, 226. [CrossRef]

15. Hui-Yin, Y.; Ahmad, N.; Azmi, N.; Makmor-Bakry, M. Curcumin: The molecular mechanisms of action in inflammation and cell death during kainate-induced epileptogenesis. Indian J. Pharm. Edu. Res. 2018, 52, 32-41. [CrossRef]

16. Hake, I.; Schönenberger, S.; Neumann, J.; Franke, K.; Paulsen-Merker, K.; Reymann, K.; Ismail, G.; Bin Din, L.; Said, I.M.; Latiff, A.; et al. Neuroprotection and enhanced neurogenesis by extract from the tropical plant Knema laurina after inflammatory damage in living brain tissue. J. Neuroimmunol. 2009, 206, 91-99. [CrossRef]

17. Cherry, J.D.; Olschowka, J.A.; O'Banion, M.K. Neuroinflammation and $M_{2}$ microglia: The good, the bad, and the inflamed. J. Neuroinflamm. 2014, 11, 98. [CrossRef]

18. Ma, Y.; Wang, J.; Wang, Y.; Yang, G.Y. The biphasic function of microglia in ischemic stroke. Prog. Neurobiol. 2016, 157, 247-272. [CrossRef]

19. Choi, J.Y.; Kim, J.Y.; Kim, J.Y.; Park, J.; Lee, W.T.; Lee, J.E. $M_{2}$ phenotype microglia-derived cytokine stimulates proliferation and neuronal differentiation of endogenous stem cells in ischemic brain. Exp. Neurobiol. 2017, 26, 33-41. [CrossRef]

20. Ahn, S.; Joyner, A. In vivo analysis of quiescent adult neural stem cells responding to Sonic hedgehog. Nature 2005, 437, 894-897. [CrossRef]

21. Bauer, S. Cytokine control of adult neural stem cells. Ann. N. Y. Acad. Sci. 2009, 1153, 48-56. [CrossRef] [PubMed]

22. Vieira, M.S.; Santos, A.K.; Vasconcellos, R.; Goulart, V.A.; Parreira, R.C.; Kihara, A.H.; Ulrich, H.; Resende, R.R. Neural stem cell differentiation into mature neurons: Mechanisms of regulation and biotechnological applications. Biotechnol. Adv. 2018, 7, 1946-1970. [CrossRef] [PubMed]

23. Martínez-Garza, D.M.; Cantú-Rodríguez, O.G.; Jaime-Pérez, J.C.; Gutiérrez-Aguirre, C.H.; Góngora-Rivera, J.F.; Gómez-Almaguer, D. Current state and perspectives of stem cell therapy for stroke. Med. Univ. 2016, 72, 169-180. [CrossRef]

24. Matarredona, E.R.; Talaveron, R.; Pastor, A.M. Interactions between neural progenitor cells and microglia in the subventricular zone: Physiological implications in the neurogenic niche and after implantation in the injured brain. Front. Cell. Neurosci. 2018, 12, 268. [CrossRef]

25. Zhang, Z.; Chopp, M. Neural stem cells and ischemic brain. J. Stroke 2016, 18, 267-272. [CrossRef] [PubMed] 
26. Faiz, M.; Sachewsky, N.; Gascón, S.; Bang, K.A.; Morshead, C.M.; Nagy, A. Adult neural stem cells from the subventricular zone give rise to reactive astrocytes in the cortex after stroke. Cell Stem Cell 2015, 17, 624-634. [CrossRef] [PubMed]

27. Choi, Y.S.; Lee, M.Y.; Sung, K.W.; Jeong, S.W.; Choi, J.S.; Park, H.J.; Kim, O.N.; Lee, S.B.; Kim, S.Y. Regional differences in enhanced neurogenesis in the dentate gyrus of adult rats after transient forebrain ischemia. Mol. Cells 2003, 16, 232-238.

28. Barkho, B.Z.; Zhao, X. Adult neural stem cells: Response to stroke injury and potential for therapeutic applications. Curr. Stem Cell Res. Ther. 2011, 6, 327-338. [CrossRef]

29. Ariffin, S.H.Z.; Wahab, R.M.A.; Ismail, I.; Mahadi, N.M.; Ariffin, Z.Z. Stem cells, cytokines and their receptors. Asia-Pac. J. Mol. Biol. Biotechnol. 2005, 13,1-13.

30. Lira-Diaz, E; Gonzalez-Perez, O. Emerging roles of microglia cells in the regulation of adult neural stem cells. Neuroimmunol. Neuroinflamm. 2008, 3, 204-206.

31. Boese, A.C.; Le, Q.S.E.; Pham, D.; Hamblin, M.H.; Lee, J.P. Neural stem cell therapy for subacute and chronic ischemic stroke. Stem Cell Res. Ther. 2018, 9. [CrossRef] [PubMed]

32. Reynolds, A.; Rubin, J.; Clermont, G.; Day, J.; Vodovotz, Y.; Bard Ermentrout, G. A reduced mathematical model of the acute inflammatory response: I. derivation of model and analysis of anti-inflammation. J. Theor. Biol. 2006, 242, 220-236. [CrossRef] [PubMed]

33. Kumar, R.; Clermont, G.; Vodovotz, Y.; Chow, C.C. The dynamics of acute inflammation. J. Theor. Biol. 2004, 230, 145-155. [CrossRef] [PubMed]

34. Alharbi, S.; Rambely, A. A dynamic simulation of the immune system response to inhibit and eliminate abnormal cells. Symmetry 2019, 11, 572. [CrossRef]

35. Hao, W.; Friedman, A. Mathematical model on Alzheimer'sdisease. BMC Syst. Biol. 2016, 10, 108. [CrossRef]

36. Nakata, Y.; Getto, P.; Marciniak-Czochra, A.; Alarcón, T. Stability analysis of multi-compartment models for cell production systems. J. Biol. Dyn. 2012, 6, 2-18. [CrossRef]

37. Ziebell, F.; Martin-Villalba, A.; Marciniak-Czochra, A. Mathematical modelling of adult hippocampal neurogenesis: Effects of altered stem cell dynamics on cell counts and bromodeoxyuridine-labelled cells. J. R. Soc. Interface 2014, 11, 20140144. [CrossRef]

38. Cacao, E.; Cucinotta, F.A. Modeling impaired hippocampal neurogenesis after radiation exposure. Radiat. Res 2016, 185, 319-331. [CrossRef]

39. Huang, L.; Zhang, L. Neural stem cell therapies and hypoxic-ischemic brain injury. Prog. Neurobiol. $2018,173$. [CrossRef]

40. Leah, E.V.; Prerna, R.R.; Raj, G.K.; Amy, K.; Jonathan, E.R. A mathematical model of neuroinflammation in severe clinical traumatic brain injury. J. Neuroinflamm. 2018, 15, 345. [CrossRef]

41. Zhao, L.; Zhang, J. Cellular Therapy for Stroke and CNS Injuries; Springer Series in Translational Stroke Research; Springer: Berlin, Germany, 2015; pp. 34-39.

42. Loane, D.J.; Kumar, A. Microglia in the TBI brain: The good, the bad, and the dysregulated. Exp. Neurol. 2016, 275, 316-327. [CrossRef] [PubMed]

43. Kim, J.Y.; Kim, N.; Yenari, M.A. Mechanisms and potential therapeutic applications of microglial activation after brain injury. CNS Neurosci. Ther. 2015, 21, 309-319. [CrossRef] [PubMed]

44. Saha Ray, S.; Sahoo, S. Generalized Fractional Order Differential Equations Arising in Physical Models; CRC Press: Boca Raton, FL, USA, 2018.

45. Gantmacher, F.R. The Theory of Matrices; American Mathematical Society: Providence, RI, USA, 1959; Volume 2.

(C) 2020 by the authors. Licensee MDPI, Basel, Switzerland. This article is an open access article distributed under the terms and conditions of the Creative Commons Attribution (CC BY) license (http:/ / creativecommons.org/licenses/by/4.0/). 